\title{
Modeling gas flow through microchannels and nanopores
}

\author{
Subrata Roya) and Reni Raju \\ Computational Plasma Dynamics Laboratory, Department of Mechanical Engineering Kettering University, \\ Flint, Michigan 48504 \\ Helen F. Chuang, ${ }^{\text {b) }}$ Brett A. Cruden, ${ }^{\text {c) }}$ and M. Meyyappan \\ Center for Nanotechnology, NASA Ames Research Center, Moffett Field, California 94035
}

(Received 22 November 2002; accepted 21 January 2003)

\begin{abstract}
Microchannel based systems have emerged as a critical design trend in development of precise control and maneuvering of small devices. In microelectronics, space propulsion and biomedical areas, these systems are especially useful. Nanoscale pores are recently becoming of great interest due to their beneficial drag and heat transfer properties. However it is difficult to predict the flow performance of these microsystems and nanosystems numerically since the standard assumptions of using Navier-Stokes equations break down at micrometer scales, while the computational times of applicable molecular-dynamics codes become exorbitant. A two-dimensional finite-element based microscale flow model is developed to efficiently predict the overall flow characteristics up to the transition regime for reasonably high Knudsen number flow inside microchannels and nanopores. Presented two-dimensional numerical results for Poiseuille flow of a simple fluid through the microchannel are comparable to the numerical and experimental data published in the literature. The nanopore solutions are also validated with presented experimental data. (C) 2003 American Institute of Physics. [DOI: 10.1063/1.1559936]
\end{abstract}

\section{INTRODUCTION}

Recently noticeable progress has been made in the field of microelectromechanical systems (MEMS). A wide variety of microscale devices like sensors, actuators, and valves are now extensively used in our everyday life. Some MEMS devices have also been designed in the field of fluid applications. Examples include micropumps, microvalves, and microturbines. A parallel revolution in nanotechnology is becoming the fastest growing research focus in the engineering community. It combines diverse fields like molecular biology, particle physics, space propulsion, and microelectronics. Understanding the interactions between and within molecules is fundamental to this development of nanoscale devices. Flows inside nanoscale pores are also important due to their highly beneficial drag and heat transfer properties.

Consequently, these emerging fields have propelled strong interest in understanding the physical aspects of these small systems and the physical laws governing them. One of the basic steps in understanding these processes is the experimental and numerical study of fluid flow through microscale channels, now commonly referred to as microchannels. In general, fluid flow through microchannels is modeled using either the continuum or the molecular approach. ${ }^{1-4}$ The continuum approach solves for macroscopic fluid properties (dependent variables) as a function of the spatial coordinates (independent variables) and is used widely for fluid flow applications. As the length scale of a physical system de-

\footnotetext{
a) Electronic mail: sroy@kettering.edu

b) Intern through NASA-USRP program, presently senior at California Institute of Technology.

${ }^{c}$ Employed through contract to Eloret Corporation.
}

creases, the validity of the standard continuum approach with no-slip boundary conditions diminishes. A widely recognized dimensionless parameter that determines the degree of appropriateness of the continuum model is Knudsen number $\mathrm{Kn}$. It is defined as the ratio of the fluid mean-free-path $\lambda$ and the macroscopic length scale of the physical system $\Lambda$.

$$
\mathrm{Kn}=\frac{\lambda}{\Lambda},
$$

where $\lambda=16 \mu / 5 \rho \sqrt{2 \pi R T}$, using the Chapman-Enskog result for the coefficient of dynamic viscosity $\mu$ in a hard sphere gas at temperature $T$, and $\Lambda=\rho / \partial \rho / \partial x$, where $\rho$ is the fluid density. The Knudsen number can thus be interpreted based on the ratio between the Mach number Ma $=u / \sqrt{\gamma R T}$ and the Reynolds number $\operatorname{Re}=\rho u D / \mu$ as,

$$
\mathrm{Kn}=\sqrt{\frac{\pi \gamma}{2}} \frac{\mathrm{Ma}}{\operatorname{Re}}=\sqrt{\frac{\pi}{2 R T}} \frac{\mu}{\rho D},
$$

where $\gamma$ is the specific heat ratio, $u$ is the fluid velocity, $R$ is the specific gas constant, and $D$ is the characteristic dimension of the system.

The local Knudsen number is a measure of the degree of rarefaction of gases encountered in small flows through narrow channels. Figure 1 describes different regimes of fluid flow depending on the Knudsen number. As Kn increases, the rarefaction effects become more pronounced and eventually the continuum assumption breaks down.

An alternative to continuum is the molecular model, ${ }^{5-7}$ which recognizes the fluid as a swarm of discrete particles. In this model the position, inertia, and state of all individual particles are calculated either deterministically ${ }^{8-10}$ or probabilistically ${ }^{3,11-13}$ at all times. The molecular approach 


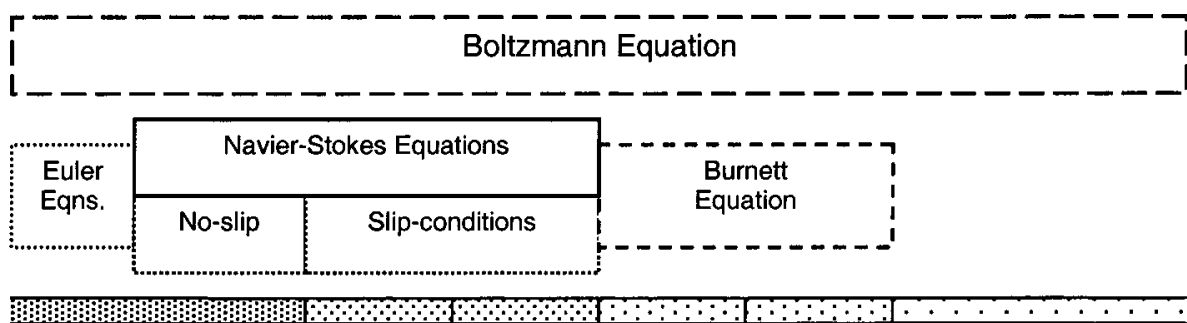

FIG. 1. Knudsen number regimes.

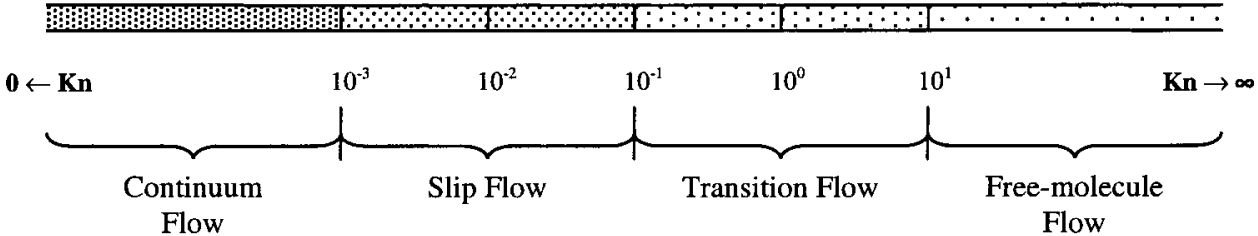

considers individual particle dynamics based on a Boltzmann distribution at the temperature of interest. The interaction between two particles is given by the two-body potential energy and transient evolution of particle positions are determined by numerically integrating the Newton's equations of motion. The choice of potential for particular fluid and solid combinations is ad hoc. From the documented contributions in the literature, ${ }^{1-2,7-10}$ it is evident that despite the theoretical appropriateness of molecular-dynamics (MD) models for the purpose of capturing the small-scale interactions, it is unrealistic to simulate a reasonably practical microflow problem using the present-day supercomputers. Most MD calculations are restricted to femtoseconds time steps limiting the results to short time scale (picoseconds to nanoseconds) phenomena. ${ }^{8-10}$ Gad-el-hak ${ }^{2}$ cites an example of $1 s$ real-time simulation for complex molecular interaction using molecular dynamics that estimates thousands of years of CPU time requirement.

For dilute gas, molecular dynamics becomes highly inefficient while microflow analysis using probabilistic models like direct simulation Monte Carlo (DSMC) provides an accurate solution as the time step diminishes and the number of particles in the cell approaches a high number. However, the error in DSMC is inversely proportional to the square root of the number of simulated molecules ${ }^{14}$ and thus decreasing the number of particles in the computational cell drastically increases the solution error. ${ }^{15}$ The cell size should be one-third of the local mean free path, otherwise it leads to error in calculation of the dynamic viscosity coefficients. ${ }^{14}$ Both molecular dynamics and DSMC have very slow convergence rates compared to continuum models. Also, due to the time splitting of the molecular motion and collisions, the maximum allowable DSMC time step needs to be smaller than the collisional time scale. For a practical problem involving a large matrix of candidate particles, these numerical restrictions translate to prohibitively expensive computational resources, severely limiting the application of DSMC to smallscale problems.

As a viable remedy, several recent publications ${ }^{16-18}$ have applied the Burnett equation ${ }^{19}$ for rarified gas flows. These continuum equation solutions are reasonably fast compared to the molecular approach. However, accurate application of the Burnett equations becomes complicated as the Knudsen number increases beyond the slip flow regime and the solu- tion becomes unstable due to small wavelength disturbances. Since Burnett equations violate the entropy condition of thermodynamics at high $\mathrm{Kn}$, artificial regularization becomes necessary to stabilize the solution. ${ }^{20}$ Recently, Sokhan et $a .^{21}$ have presented hydrodynamic boundary conditions for nonequilibrium molecular dynamics (NEMD) solutions in a $7.1 \mathrm{~nm}$ carbon nanopore. The relaxation times in NEMD, which are of the order of several picoseconds for Poiseuille flow of simple uniform fluids, increase by several orders of magnitude in confined geometries. The results in Ref. 21 show that in order to capture important fluctuations in collective physical flow properties an integration time of the order of $100 \mu \mathrm{s}$ is essential while an equilibration time of the order of $1 \mathrm{~ns}$ is required for their computational domain.

As a means of achieving numerically efficient microflow and nanoflow predictions with reasonable computational time, in this article we develop, implement, and document the performance of a finite element discretized Galerkin weak statement based phase space model for small flow applications. We used sufficiently wide pores of up to $200 \mathrm{~nm}$ diameter for which the boundary layers of fluid having modified structure do not overlap. The hydrodynamic description of the fluid flow is reasonable for this case. ${ }^{22}$ The aim of this numerical study is an exploratory prediction of the overall flow characteristics of two practical multidimensional microsystems and nanosystems within and beyond the slip flow regime.

This article is organized into the following sections. Section I is a brief introduction of the state of the art. Section II provides a general description of the multidimensional microchannel and nanopore flow problems. Section III states the governing flow equations. The development of weak statement algebra into finite element iterative algorithm is documented in Sec. IV. Relevant boundary conditions for both microscale and nanoscale flow simulation are given in Sec. V. Section VI is divided into two distinct parts. First, the numerical results for the microchannel flow are benchmarked using experimental data ${ }^{23}$ and numerical results ${ }^{24}$ reported in the literature. Then, the nanopore experimental data are presented and compared with the two-dimensional finite element numerical model. 


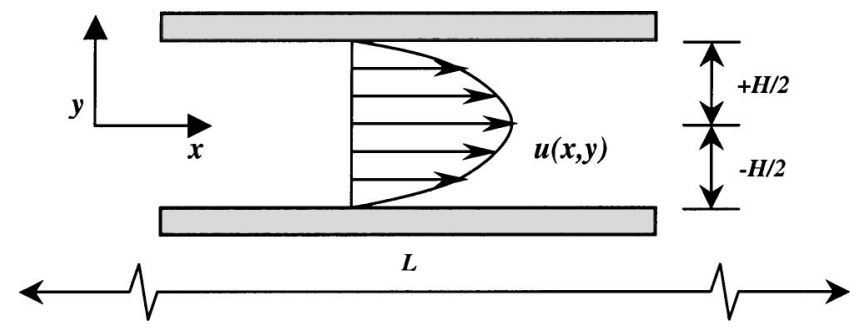

FIG. 2. Geometry for microchannel analysis.

\section{MODEL DESCRIPTION}

\section{A. Microchannel}

The cross section of the microchannel is shown in Fig. 2. Several experimental and numerical results have been documented for this popular geometry. Pfahler et al..$^{25}$ and Choi et $a l .{ }^{26}$ studied the friction factor for the flow through microtubes. Harley ${ }^{27}$ has conducted both the numerical and experimental study of the microchannel flow; however the numerical model has employed general no-slip assumption. Arkilic ${ }^{28}$ conducted an experimental study of flow through microchannels and also the effect of tangential momentum accommodation coefficients for slip flow conditions. Pong et $a l .{ }^{23}$ have experimentally investigated the nonlinear distribution of pressure along a microchannel. Chen et al. ${ }^{24}$ developed a finite difference formulation for slip flow and compared their results with experiment. ${ }^{23}$

In this article, we validate the numerical results for gas flow through the microchannel with the reported experimental data of Pong et al. ${ }^{23}$ The flow simulation is also benchmarked for five different pressure ratios using the numerical results of Chen et al. ${ }^{24}$ Following the experimental and the numerical study the exit pressure is fixed to be $100.8 \mathrm{kPa}$ and the Knudsen number at the outlet is 0.058 , which is well within the slip flow regime. Table I lists the dimensions of the microchannel and relevant physical properties of the working fluid nitrogen.

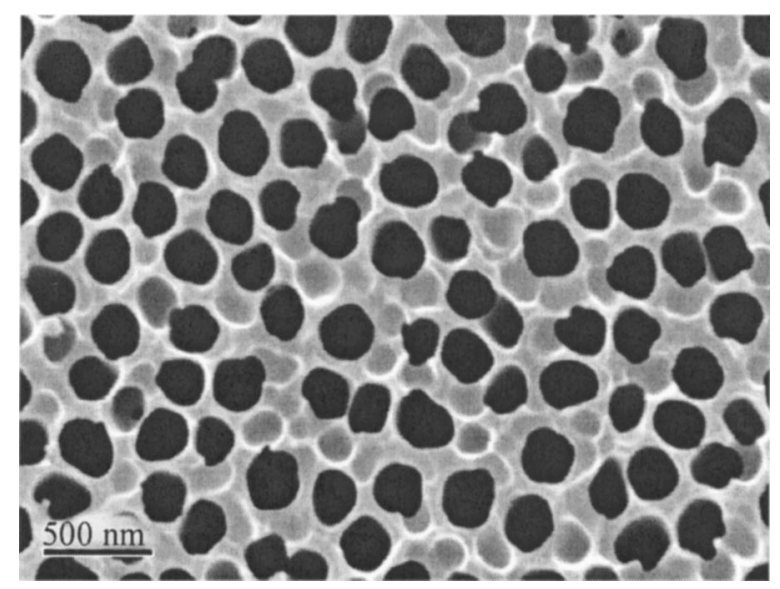

FIG. 3. SEM image of anodisc membrane.

\section{B. Nanopore}

Instrumentation for nanoscale measurements to date has been very difficult and, sometimes, imprecise. Consequently, nanopore flow data is relatively scarce. The experimental data for this article were collected from commercial Whatman alumina filters (Anodisc 13) with pore sizes of $200 \mathrm{~nm}$ and a thickness of $60 \mu \mathrm{m}$. The density and sizes of pores were measured in a scanning electron microscope (SEM) and found to be $5-8 \times 10^{12} \mathrm{~m}^{-2}$ and $212 \mathrm{~nm}$, respectively, giving an estimated porosity of $0.2-0.3$. The SEM image of the surface of these filters is shown in Fig. 3. The nominal dimension of a single nanopore and the physical properties of the working fluid are given in Table I for pressure ranging between 0-1200 torr. Computational studies involving molecular dynamics, kinetic Monte Carlo, and lattice gas model for nanoscale bounded flows exhibit diffusive characteristic of normal, single-file, and transition mode. ${ }^{9,10,29-31}$ The distinction between normal and single-file modes, however, only exists when studying the transport of individual molecules. The bulk flow and transport characteristics cannot be

TABLE I. Model dimensions and fluid properties.

\begin{tabular}{lcc}
\hline \hline Flow parameters & Microchannel & Nanopore \\
\hline Length $L$ & $3000 \mu$ & $60 \mu$ \\
Width $W$ & $40 \mu$ & \\
Height $H$ & $1.2 \mu$ \\
Diameter $D$ & & $200 \mathrm{~nm}$ \\
Pressure ratio $P_{r}=P_{\text {in }} / P_{\text {out }}$ & $1.34,1.68,2.02,2.36,2.70$ & \\
Pressure difference $\Delta P=P_{\text {in }}-P_{\text {out }}$ & & $100.0,200.0,300.0,400.0,500.0$, \\
& $100.8 \mathrm{kPa}$ & $600.0,750.0,900.0$ torr \\
Outlet pressure $P_{\text {out }}$ & $314 \mathrm{~K}$ & $4.8 \mathrm{kPa}$ \\
Temperature at the inlet $T_{i}$ & $314 \mathrm{~K}$ & $300 \mathrm{~K}$ \\
Wall Temperature $T_{w}$ & 0.058 & $300 \mathrm{~K}$ \\
Exit Knudsen number $K$ n & $1.85 \times 10^{-5} \mathrm{Ns} / \mathrm{m}^{2}$ & 7.36 \\
Absolute viscosity $\mu$ & $296.7 \mathrm{~J} / \mathrm{kg} \mathrm{K}$ & $2.22 \times 10^{-5} \mathrm{Ns} / \mathrm{m}^{2}$ \\
Specific gas constant $R$ & 1.4 & $208.1 \mathrm{~J} / \mathrm{kg} \mathrm{K}$ \\
Ratio of specific heats $\gamma$ & $28.013 \mathrm{~kg} / \mathrm{kmol}$ & 1.667 \\
Molecular mass & & $39.948 \mathrm{~kg} / \mathrm{kmol}$ \\
\hline \hline
\end{tabular}


distinguished for systems displaying single-file or normal diffusion. ${ }^{32}$ Thus, we are not in a position to distinguish between these modes of transport in this work.

\section{GOVERNING EQUATIONS}

The Knudsen number for our particular problem of interest ranges from slip (0.058) to transition (7.36) regime. We shall use the governing equations of standard NavierStokes (NS) in two dimensions. ${ }^{2}$ The "no-slip" wall condition in the usual continuum description is defined as having all components of the velocity vanish at the solid wall. However, as the macroscopic length scale becomes comparable to the fluid mean free path, the description becomes molecular. Therefore, the streaming velocity at the wall comprises of the streaming velocity of incident particles and that of the scattered particles. The boundary condition in this case can be interpreted as the flux or Neumann condition from the macroscopic point of view. One may, of course, use a Taylorseries expansion on mean free path (Knudsen number) to determine the wall streaming velocity as a function of normal derivatives. Maxwell ${ }^{33}$ derived the first-order slip relations for dilute, monatomic gases. We shall implement firstorder slip boundary conditions in the momentum and energy equations for $10^{-3} \leqslant \mathrm{Kn} \leqslant 10$.

The wall-slip boundary condition for an ideal gas is given as,

$$
u_{\mathrm{gas}}-u_{\mathrm{wall}}=\frac{2-\sigma_{V}}{\sigma_{V}} \lambda\left(\frac{\partial u}{\partial y}\right)_{w}+\frac{3}{4} \frac{\mu}{\rho T_{\mathrm{gas}}}\left(\frac{\partial T}{\partial x}\right)_{w} .
$$

The corresponding temperature-jump relation was derived by von Smoluchowski ${ }^{34}$ as

$$
T_{\text {gas }}-T_{\text {wall }}=\frac{2-\sigma_{T}}{\sigma_{T}}\left[\frac{2 \gamma}{\gamma+1}\right] \frac{\lambda}{\operatorname{Pr}}\left(\frac{\partial T}{\partial y}\right)_{w} .
$$

In Eqs. (3a)-(3b), $u_{\text {gas }}$ and $T_{\text {gas }}$ are the velocity and temperature of the gas adjacent to the wall, while $u_{\text {wall }}$ and $T_{\text {wall }}$ are the wall velocity and wall temperature, respectively, Pr is the nondimensional Prandtl number, and the subscript $w$ denotes wall condition. The second term in Eq. (3a) is known as "thermal creep," which generates slip velocity in the direction opposite to the increasing temperature.

The slip-wall conditions $(3 a)-(3 b)$ use the tangentialmomentum accommodation coefficient $\sigma_{v}$ and the thermal accommodation coefficient $\sigma_{T}$ at the walls. These coefficients indicate the fraction of the molecules reflected diffusively from the walls. For example, for $\sigma_{v}=0$ the molecules reflect specularly indicating the reversal in their normal velocity due to normal momentum transfer to the wall. For $\sigma_{v}=1$ the molecules reflect diffusively when reflected from the wall with zero tangential velocity. The value of the coefficients $\sigma_{V}$ and $\sigma_{T}$ depends on the surface finish, the fluid, temperature, and local pressure. The value of $\sigma_{V \approx 0.80}$ for nitrogen, argon, or carbon dioxide in a silicon micromachined channel has been determined experimentally by Arkilic. $^{28}$

The dimensionless form of Eqs. (3a) and (3b) are given by Gad-el-Hak, ${ }^{2}$

$$
\begin{aligned}
u_{\mathrm{gas}}^{*}-u_{\mathrm{wall}}^{*}= & \frac{2-\sigma_{V}}{\sigma_{V}} \operatorname{Kn}\left(\frac{\partial u^{*}}{\partial y^{*}}\right)_{w} \\
& +\frac{3}{2 \pi}\left(\frac{\gamma-1}{\gamma}\right) \frac{\mathrm{Kn}^{2} \operatorname{Re}}{\mathrm{Ec}}\left(\frac{\partial T^{*}}{\partial x^{*}}\right)_{w}, \\
T_{\mathrm{gas}}^{*}-T_{\mathrm{wall}}^{*}= & \frac{2-\sigma_{T}}{\sigma_{T}}\left[\frac{2 \gamma}{\gamma+1}\right] \frac{\mathrm{Kn}}{\operatorname{Pr}}\left(\frac{\partial T^{*}}{\partial y^{*}}\right)_{w},
\end{aligned}
$$

where Re and Ec are the nondimensional Reynolds and Eckert number, respectively.

Note that traditionally the first-order slip conditions are applicable to Navier-Stokes equations when the $\mathrm{Kn}$ is in the range of $0.001-0.1$. For $\mathrm{Kn}>0.1$, documented literatures ${ }^{2,14,35}$ find it necessary to develop higher-order slip velocity conditions as the continuum regime breaks down. A higher-order slip velocity is given by Karniadakis and Beskok $^{2,35}$

$$
\begin{aligned}
u_{\mathrm{gas}}^{*}-u_{\mathrm{wall}}^{*}= & \frac{2-\sigma_{V}}{\sigma_{V}} \frac{\mathrm{Kn}}{1-b \mathrm{Kn}}\left(\frac{\partial u^{*}}{\partial y^{*}}\right)_{w} \\
& +\frac{3}{2 \pi}\left(\frac{\gamma-1}{\gamma}\right) \frac{\mathrm{Kn}^{2} \operatorname{Re}}{\mathrm{Ec}}\left(\frac{\partial T^{*}}{\partial x^{*}}\right)_{w},
\end{aligned}
$$

where $b(\mathrm{Kn})$ is an empirical parameter determined analytically for the slip flow regime as $\left.b=1 / 2(\partial \omega / \partial n / \omega)_{w}\right)$, the ratio of the vorticity flux to the wall vorticity, and by an empirical curve fitting using the solutions of the corresponding NS and DSMC numerical model to a high order accurate in $\mathrm{Kn}$ for the transition regime. For the microchannel case in our study, Kn remains within 0.058, however for the nanopore $\mathrm{Kn}$ increases up to 7.36. Contrary to common practice, we would restrict slip to first-order conditions (4)-(5) for both the microchannel and the nanopore.

\section{NUMERICAL METHOD}

Finite element algorithms are used extensively in numerical modeling of fluid flow and heat transfer. ${ }^{36,37} \mathrm{Re}$ cently, Roy and Pandey have implemented it for analyzing the partially ionized gas flow inside a Hall thruster. ${ }^{38,39}$ Although continuous basis function based finite elements are limited to larger length scale and may not be accurate for $\sim 1$ $\mathrm{nm}$ or smaller size pores, recent developments in local discontinuous Galerkin $^{40}$ shows promise for nanoporous applications where quantum effects become important. In this article, we extend the finite element formulation ${ }^{37-39}$ to predict the fluid flow through the microchannel and sufficiently wide (200 nm) nanopore. The choice of time step is dictated by the Courant-Fredrich-Levy condition. ${ }^{41}$ The code uses fully implicit variable time steps until the transient features die down as the Newton-Raphson iteration converges to a steady state. The solution is declared convergent when the maximum residual for each of the state variables becomes smaller than a chosen convergence criterion of $\in=10^{-4}$. Here, the convergence of a solution vector $\mathrm{U}$ on node $j$ is defined as the norm: $\left\|\mathbf{U}_{j}-\mathbf{U}_{j-1}\right\| /\left\|\mathbf{U}_{j}\right\| \leqslant \in$. The governing equations are solved 
using a fully implicit numerical procedure. The computational channel (pore) geometry is discretized using twodimensional nine-noded biquadratic finite elements. The continuity and equation of state are solved for pressure and density, respectively, using the four corner nodes of the element. For velocity and temperature calculations, all nine nodes of the biquadratic element are used.

Table I describes the inlet, outlet, and wall condition details for the microchannel and nanopore. At the inlet the gas temperature $T_{i}$ is specified as $314 \mathrm{~K}$ and based on the assumption of the isothermal wall, the wall temperature $T_{w}$ is also specified as $314 \mathrm{~K}$. The velocity flux $\partial u / \partial x=0$ and the y component of the velocity $v=0$ at the inlet. The pressure at the outlet, $P_{0}$ is 100.8 for microchannel $(4.8 \mathrm{kPa}$ for nanopore) and the inlet pressure, $P_{i}$ is specified based on the corresponding pressure ratio (microchannel) or pressure drop (nanopore). The microchannel is benchmarked using both no-slip and first-order slip condition. The nanopore solution is validated using first-order slip condition. For both cases, we assume $\sigma_{V}=\sigma_{T} \approx 1.0$ for convenience, which means that the channel surface is rough.

\section{EXPERIMENTAL PROCEDURE}

For characterization of nanoscale flows, experimental data was collected measuring flow through a porous alumina disc. The disc is a commercially available "Anodisc" membrane filter from Whatman as described above. The disc consists of an array of well-defined cylindrical pores oriented perpendicular to the membrane. Similar anodized alumina membranes have been characterized extensively in the literature. ${ }^{42,43}$ To evaluate fluid flow through these pores, an apparatus was constructed to measure pressure drop versus flow rate across the pore. The technique is analogous to the so-called manometric method. ${ }^{44,45}$ The Anodisc was mounted on strips of impermeable tape such that a well-known area in the center of the Anodisc served as a permeable area. Portions outside of this area were coated with Vac-Seal sealant and baked overnight at $60^{\circ} \mathrm{C}$ to prevent gas from diffusing between the tape/Anodisc boundary. In the manometric setup, the Anodisc is clamped between two Teflon showerheads and the pressure drop across the membrane is measured versus flow rate with an MKS differential pressure gauge (223BD) and Mass Flow Controller (M100B). The pressure drop due to the showerhead is only a few percent of that caused by the Anodisc. This relationship was measured for argon, oxygen, and nitrogen gases for pressures between $0-1200$ torr $(0-160 \mathrm{kPa})$ and fluxes between $0-9 \mathrm{~mol} / \mathrm{m}^{2} / \mathrm{s}$ $\left(0-45 \mathrm{~mol} / \mathrm{m}^{2} / \mathrm{s}\right.$ after porosity correction). The rate of depressurization of the upstream volume by transport through the Anodisc was also measured. Experimental uncertainty is determined primarily by instrument read-out errors. However, converting the macroscopically measured data to an analogous flow through a single pore is subject to converting the total flux to an effective flux per pore. This uncertainty is determined primarily by the uncertainty in the membrane porosity. Present estimates of membrane porosity from representative SEM images vary by $\pm 20 \%$.

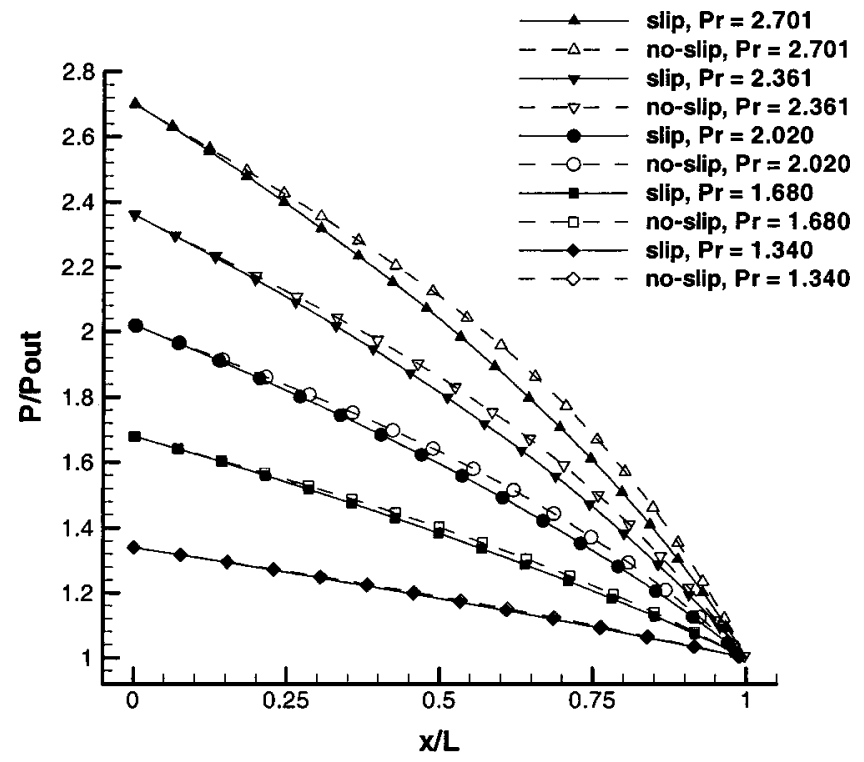

FIG. 4. Centerline pressure distribution normalized with outlet pressure $P_{\text {out }}$. Solutions are compared for the slip and the no-slip conditions for five different pressure ratios $\left(\operatorname{Pr}=P_{\text {in }} / P_{\text {out }}\right)$.

\section{RESULTS AND DISCUSSIONS}

\section{A. Microchannel Flow}

In this case, the computational geometry is discretized using 324 two-dimensional nine-noded non-overlapping biquadratic finite elements that consist of a total of 1369 nodes. The fully implicit time integration solution for the slipcorrected Navier-Stokes equations yields a nonlinear distribution of pressure, density, and velocity along the microchannel. While the roughness of the microchannel used in the experiment ${ }^{23}$ is unknown, we have used $\sigma_{v}=1$ based on the published numerical results. ${ }^{24}$ The pressure drop in the channel takes place in order to overcome the frictional forces at the walls. We note that the temperature does not change along the channel, implying that the density is directly proportional to the pressure and hence shows a drop along the channel. The velocity shows the corresponding increase to preserve the continuity equation. The increase in shear stress due to the increase in velocity causes a further drop in pressure. The effect of slip and no-slip boundary conditions on the centerline of the channel $(y=0)$ is shown in Fig. 4 . Clearly, an increase in pressure ratio shows increasing divergence in the pressure distribution between the no-slip and slip wall solutions. This effect is the most prominent for pressure ratio $P_{r}=2.701$ where the maximum percentage difference in the pressure distribution between two predictions is $\sim+4 \%$.

The corresponding velocity solutions at the channel centerline are plotted in Fig. 5. The slip condition solutions consistently show a higher magnitude of velocity than no-slip condition due to the lower-shear stress. The nonlinear distribution of the velocity is dependent on the pressure distribution and hence varies for different pressure ratios. As the pressure ratio increases, the nonlinearity of the streaming 


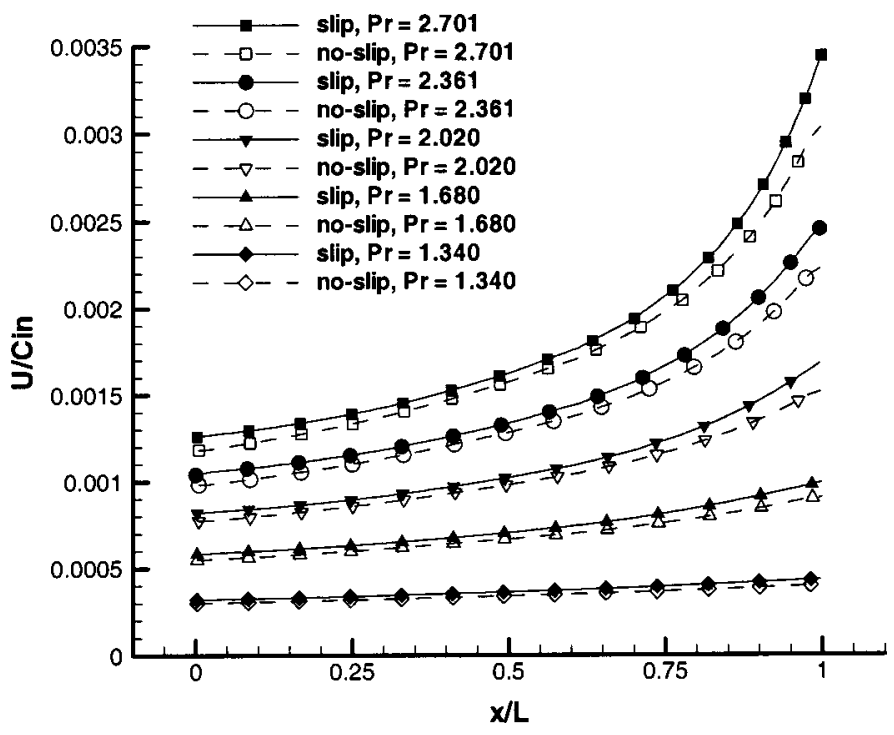

FIG. 5. Centerline velocity distribution normalized with speed of sound at the inlet $C_{\text {in }}$. Solutions are compared for the slip and the no-slip conditions the five different pressure ratios $\left(\operatorname{Pr}=P_{\text {in }} / P_{\text {out }}\right)$.

velocity distribution becomes prominent. The difference between the slip and no-slip velocity distribution shows a higher difference for the corresponding higher-pressure ratio. The percentage difference between the slip and no-slip condition is $\sim+8 \%$.

In Fig. 6, the finite element model centerline pressure results for the slip model have been validated with the experimental data of Pong et al. ${ }^{23}$ for their first generation microchannel. The experimental pressure measurements were based on four pressure sensors along the length of the channel. A commercial Omega HHP 4100 transducer was used to calibrate these sensors. Pressure signals were averaged over a period of several minutes for minimizing the $1 / f$ type flicker and popcorn noise. The maximum uncertainty for these experimental measurements was determined to be less than 1 psi (gauge) for most sensors. ${ }^{23}$ For five selected pres-

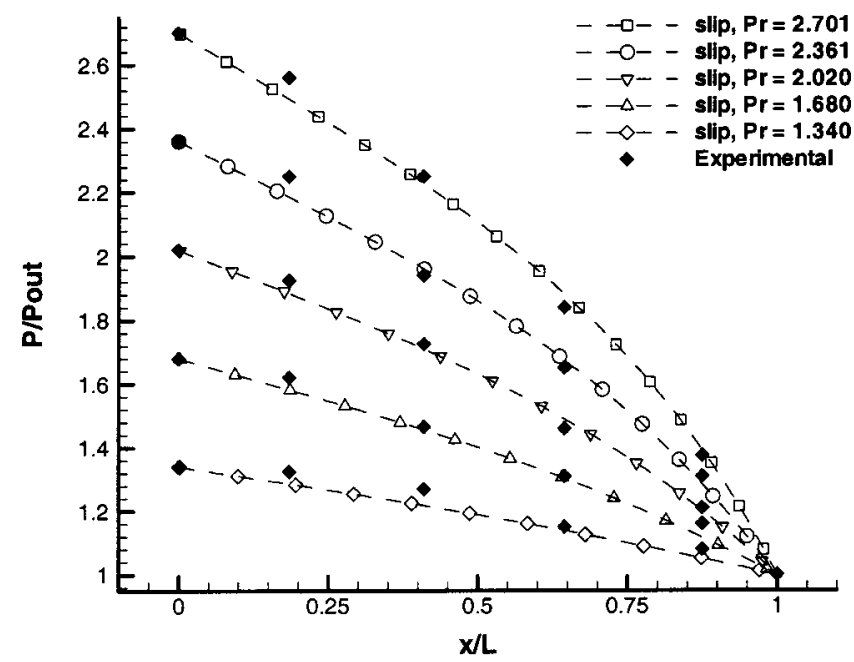

FIG. 6. Computed centerline pressure distribution normalized with outlet pressure $P_{\text {out }}$ for the slip condition using five different pressure ratios $(\mathrm{Pr}$ $\left.=P_{\text {in }} / P_{\text {out }}\right)$ are validated with experimental results (see Ref. 23).

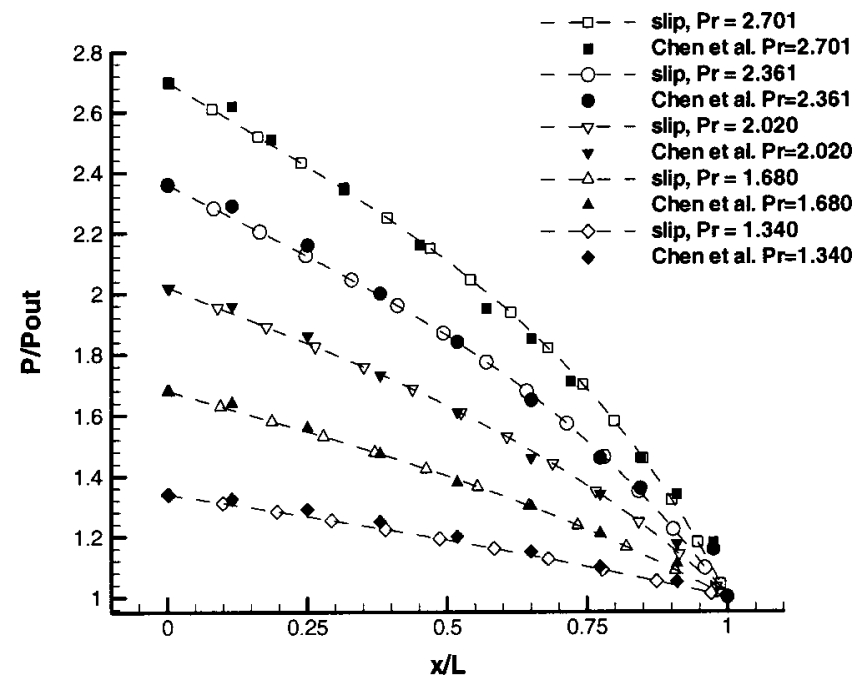

FIG. 7. Normalized pressure distribution of the finite element model solution for different pressure ratios $\left(P r=P_{\text {in }} / P_{\text {out }}\right)$ is benchmarked with reported numerical results (see Ref. 24).

sure ratios, the computed finite element model solutions are found to be within approximately $4 \%$ of the experimental data. Overall, presented simulation results underpredicted the experimental data in the upstream section and overpredicted in the downstream.

The computed solutions for the same five pressure ratios are further benchmarked with numerical results previously reported in the literature. ${ }^{24}$ Our numerical results as plotted against that of Chen et al. ${ }^{24}$ generate a similar nonlinear pressure distribution trend as shown in Fig. 7 and the centerline pressure distributions are found to be comparable within $1.3 \%$ of the reported results. It is important to note that Chen et al. utilized an explicit finite difference method on a $6000 \times 23$ grid for this simulation, while we used a mere $18 \times 18$ biquadratic finite element mesh.

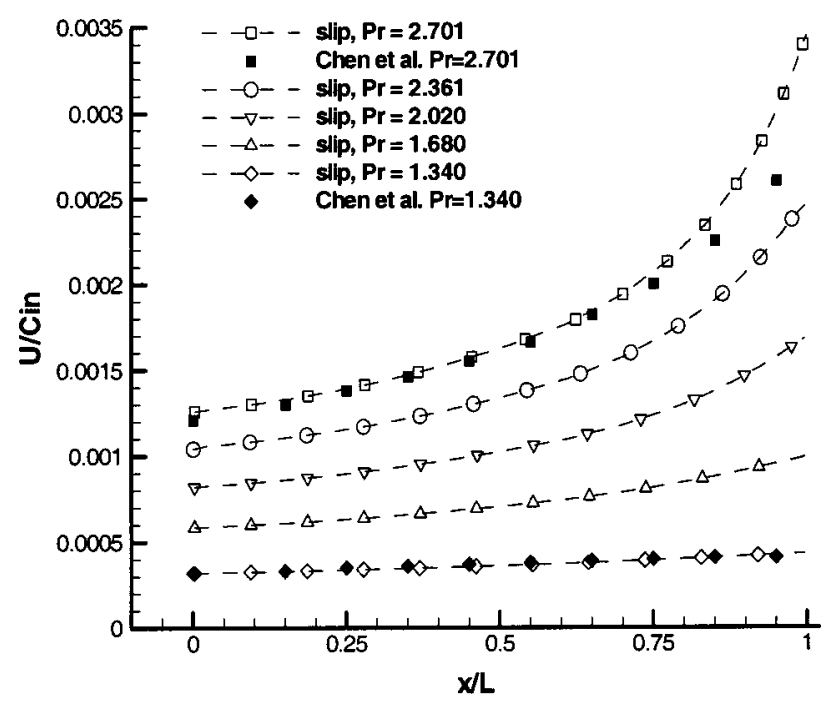

FIG. 8. Centerline velocity distribution normalized with speed of sound at the inlet $C_{\text {in }}$. The finite-element solutions for slip conditions with five different pressure ratios $\left(\operatorname{Pr}=P_{\text {in }} / P_{\text {out }}\right)$ are compared with the finite difference numerical results (see Ref. 24). 


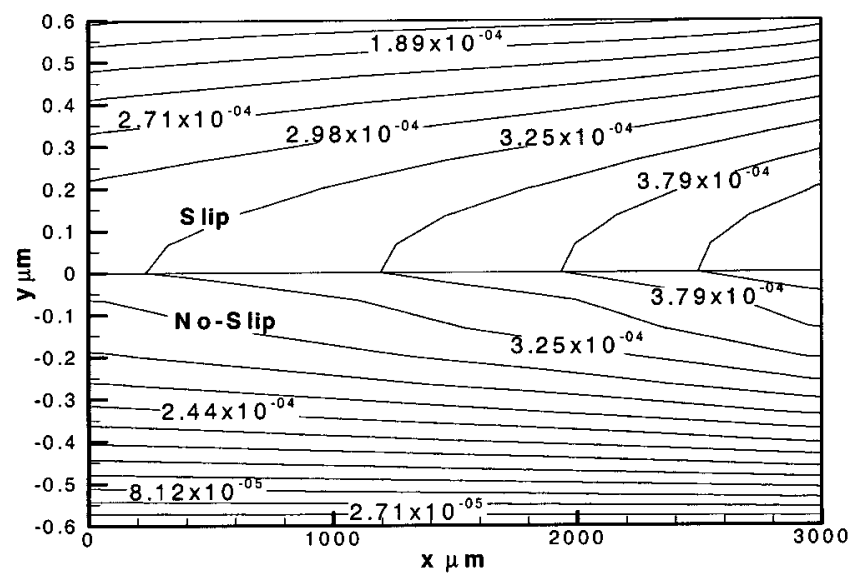

(a) $\mathrm{P}_{\mathrm{r}}=1.340$

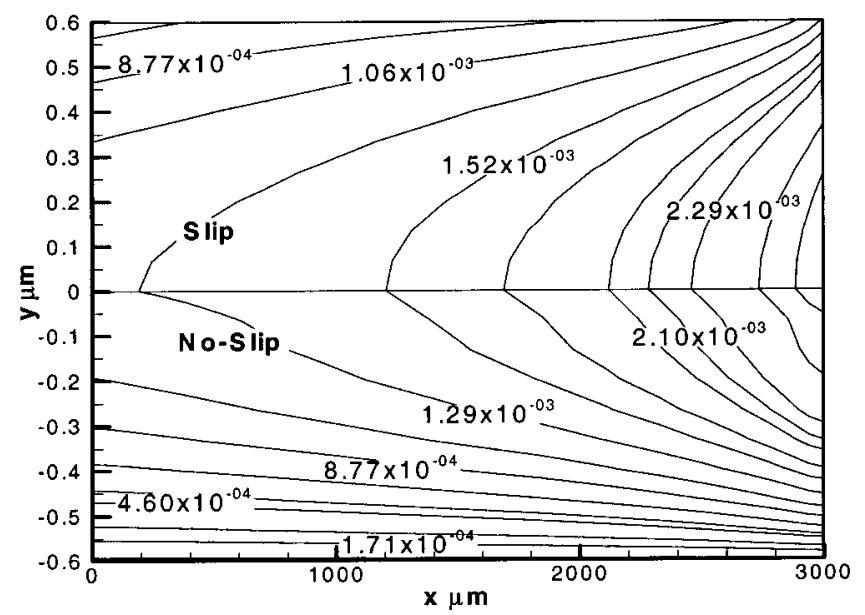

(b) $P_{r}=2.701$

FIG. 9. Two-dimensional contour plots for the streaming velocity (normalized with speed of sound at the inlet) inside the microchannel.

Finally, the slip model streaming velocity solutions along the centerline are compared with the reported numerical results ${ }^{24}$ for the highest (2.701) and lowest (1.340) pressure ratios. Experimental data was not available for this study. As evident in Fig. 8, the centerline velocity distribution of Chen et al. for both pressure ratios follow closely with our simulation results validating the computationally efficient finite element model. The 1369-node finite element result matches accurately with that of 138000 -point finite difference solution for the low-pressure ratio while for the highest-pressure ratio the finite element solution shows an $\sim 23 \%$ higher-exit velocity satisfying continuity.

The two-dimensional streaming velocity contour plots in Figs. 9(a) and 9(b) document a general description and comparison of the slip and no-slip solutions for pressure ratios 1.340 and 2.701. While the no-slip solution maintains zero velocity at the wall, the slip wall solution for 2.701 pressure ratio maintains a streaming wall velocity ranging approximately $9 \%$ (near inlet) to $15 \%$ (near exit) that of the centerline.

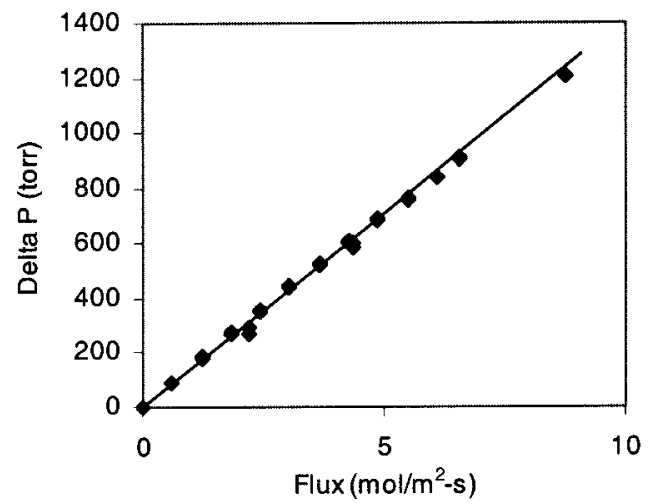

(a)

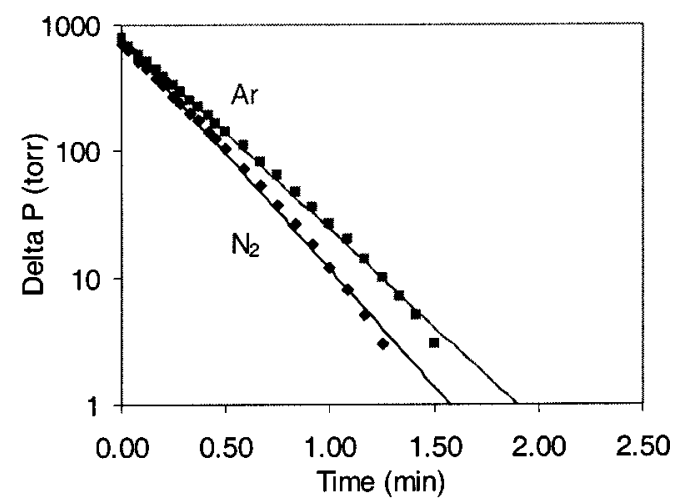

(b)

FIG. 10. (a) Plot of pressure drop versus flux for argon in an anodisc membrane. The flux is a macroscopic flux, i.e., has not been corrected for film porosity. (b) Plot of transient pressure decay for argon and nitrogen through the anodisc membrane. The line represents a best fit single exponential decay.

\section{B. Nanopore Flow}

\section{Experimental Data}

Experimental data were collected for $\mathrm{Ar}, \mathrm{N}_{2}$, and $\mathrm{O}_{2}$ flow through the nanoporous Anodisc membranes, as shown in Fig. 10. A representative steady-state data, shown in Fig. 10(a), demonstrates that the relationship between flow rate and pressure drop is linear in this regime. This is consistent with a diffusive transport regime with a constant diffusion coefficient and negligible viscous effects. Mathematically, this is expressed as

$$
\frac{F}{\epsilon A}=D_{K} \frac{\Delta P}{R T L},
$$

where $F$ is the molar flow rate, $\epsilon$ is the membrane porosity, $A$ is the exposed area, $\Delta P$ is the pressure drop across the membrane, $L$ is the thickness, $R$ is the ideal gas constant, and $T$ is the ambient temperature. In this case, we also find that the diffusion coefficient is consistent with the Knudsen diffusivity $D_{K}$ given by

$$
D_{K}=\frac{d_{\text {pore }}}{3} \sqrt{\frac{8 R T}{\pi M}}
$$

where $d_{\text {pore }}$ is the pore diameter and $M$ is the molecular weight of the diffusing species. Figure 10(b) shows transient 
TABLE II. Calculated and measured diffusivities of anodisc membrane $\left(\times 10^{-5} \mathrm{~m}^{2} / \mathrm{s}\right)$. The measured diffusivities have an accuracy of approximately $20 \%$, owing to uncertainty in the membrane porosity measurement

\begin{tabular}{lcccc}
\hline \hline & $\begin{array}{c}\text { Knudsen } \\
\text { diffusivity }\end{array}$ & $\begin{array}{c}\text { Measured } \\
\text { diffusivity }\end{array}$ & $\begin{array}{c}\text { Transient } \\
\text { diffusivity }\end{array}$ & $\begin{array}{c}\text { Calculated } \\
\text { dffusivity }\end{array}$ \\
\hline Oxygen & 3.13 & 3.01 & & \\
Nitrogen & 3.35 & 3.26 & 3.30 & \\
Argon & 2.80 & 2.66 & 2.75 & 2.79 \\
\hline \hline
\end{tabular}

data for depressurization of the apparatus. The behavior is well described by a single exponential decay, consistent with the solution to a one-dimensional transient diffusion problem

$$
\Delta p(t) \simeq p_{0} e^{-\epsilon A D_{K} / V_{u} l^{2} t},
$$

where $\Delta p(t)$ is the pressure drop across the membrane as a function of time, $p_{0}$ is the pressure drop at time $t=0$, and $V_{u}$ is the volume upstream of the membrane. Table II shows that the analytical Knudsen diffusivity is consistent with the mea-

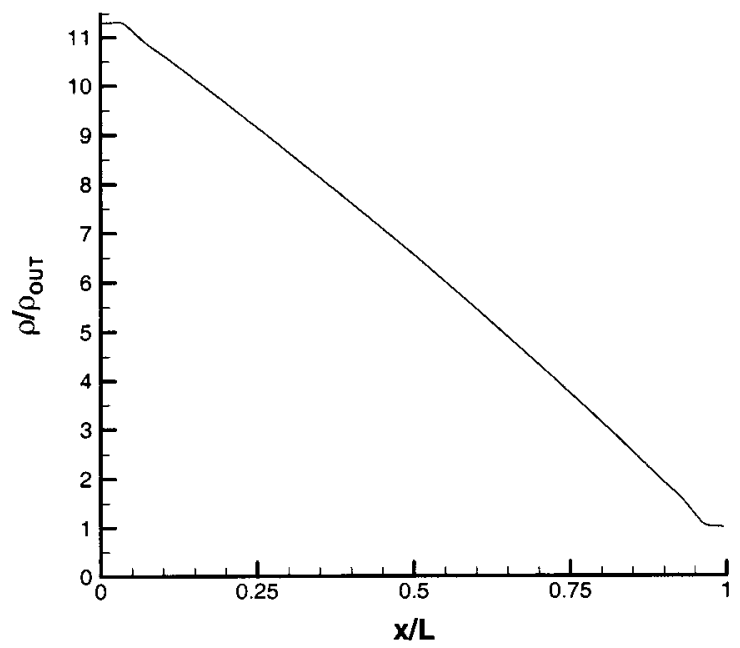

(a) sured diffusivities given the uncertainty of the porosity measurement. Given the large value of the Knudsen number under these conditions, this result is to be expected. The socalled "normal mode" diffusion would only be expected at higher-molecular densities than studied here. Still, a deviation from Knudsen diffusivity may be observed at smaller pore radii where molecule-wall interactions differ from the elastic interactions assumed in Knudsen flow. However, studies with alumina pores down to $10 \mathrm{~nm}$ in diameter still displayed Knudsen-type diffusion characteristics. ${ }^{46}$

\section{Numerical Prediction}

The nanopore geometry is discretized using $28 \times 20$ twodimensional nonoverlapping bi-quadratic finite elements resulting in a total of 2337 nodes. Here again, we utilize a fully implicit time integration and solve the dimensional NavierStokes equations using Newton-Raphson iterative procedure. The working fluid is selected to be argon. For the wall and inlet temperatures maintained at $300 \mathrm{~K}$, we assume that

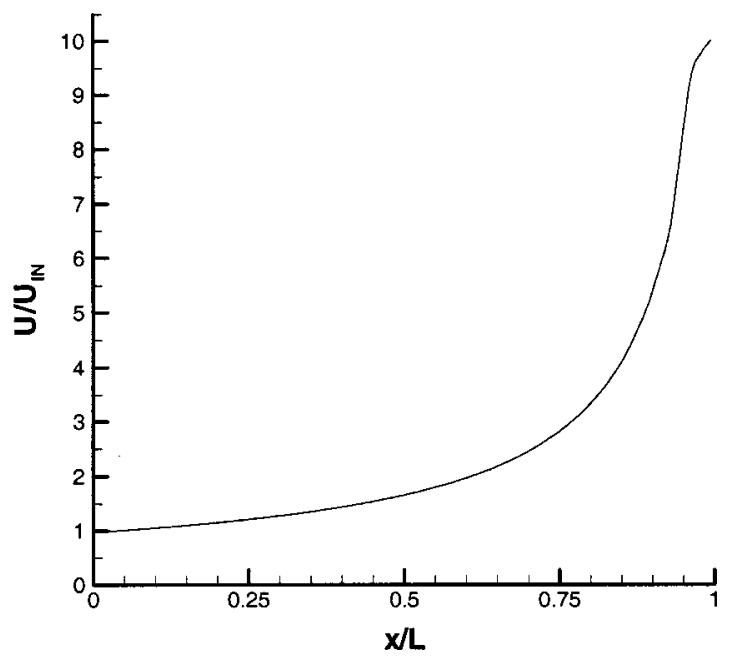

(b)

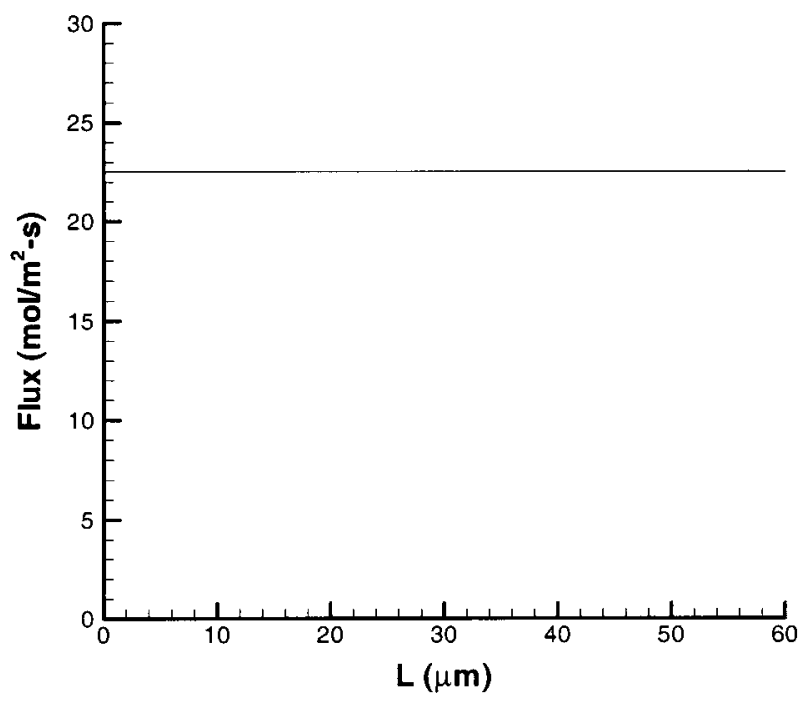

(c)

FIG. 11. (a) Density profile at the centerline, (b) velocity profile at the centerline, and (c) argon molar flux along the centerline for $\Delta P=600$ torr. 


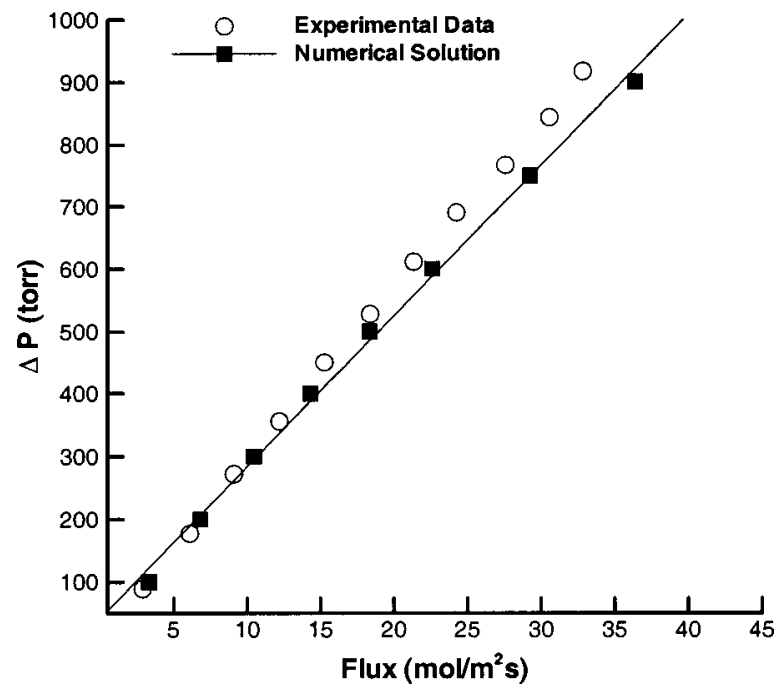

FIG. 12. Solution validation of numerical results with experimental data for argon. The line represents the best linear fit for the numerical results.

the solution temperature nearly stays isothermal along the channel, implying that the density is directly proportional to the pressure. For nanoporous alumina, Itaya et al. ${ }^{46}$ also demonstrated the linear pressure dependence of fluxes for various gases. Note that the Knudsen number for this problem reaches up to 7.36 at the outlet. Karniadakis and Beskok $^{14}$ (p. 92) has noted that for this highly rarified gas flow the pressure distribution becomes more linear. In Fig. 11 , the density, streaming velocity distribution, and the molar flux at the centerline of the pore show the presence of Knudsen diffusion regime. Solution density, see Fig. 11(a), is normalized by the outlet density while the streaming velocity in Fig. 11(b) is normalized by the inlet value. Although the roughness of the nanopore used in the experiment is unknown, it is anticipated that for a smoother wall a higher slip velocity may result in even higher-flow prediction.

Figure 12 compares the porosity corrected experimental data with the numerical prediction for argon. The numerical result deviates as the pressure drop increases. However, the linear dependence between the gas flux and the pressure drop is clearly captured. Corresponding Knudsen diffusivity is calculated for argon based on the computed molar flow rate for pressure drop across the pore as 2.79 and tabulated in Table II. The calculated diffusivity is within $+4.6 \%$ of that of the measured data and within $-0.4 \%$ of the analytical value.

\section{CONCLUSION}

A finite element based macroscopic formulation has been developed, implemented, and validated for the twodimensional flow prediction through a microchannel of aspect ratio 2500 and a nanopore of aspect ratio 300 . The results show a nonlinear distribution for pressure and velocity inside the microchannel. As the pressure ratio increases the nonlinearity grows. However, for the nanopore, a linear pressure dependence of gas flux is observed. The slip flow results for the microchannel with an outlet Knudsen number of 0.058 shows a $4 \%-8 \%$ higher-streaming velocity than the corresponding no-slip solution for the same pressure ratio. For the nanopore, the experimentally measured diffusivities for three different working fluid matches within $5 \%$ of the analytical Knudsen diffusivity. Based on the calculated flow rate for a given pressure drop, the numerically predicted diffusivity for argon is within $+4.6 \%$ of the measured data and $-0.4 \%$ of the analytical value. Although the finite element results look promising for microchannels and sufficiently wide nanopores, the efficacy at even smaller length scales needs to be explored.

\section{ACKNOWLEDGMENTS}

The authors would like to acknowledge Jeff Ifland for construction of the manometric measurement apparatus. The work of BAC is funded by NASA Ames Contract No. NAS299092 to Eloret Corporation.

${ }^{1}$ G. A. Bird, Molecular Gas Dynamics (Clarendon Press, Oxford, United Kingdom, 1976).

${ }^{2}$ M. Gad-el-hak, J. Fluids Eng. 121, 5 (1999).

${ }^{3}$ H. Xue, Q. Fan, and C. Shu, Prob. Eng. Math. 15, 213 (2000).

${ }^{4}$ D. Jie, X. Diao, K. B. Cheong, and L. K. Yong, J. Micromech. Microeng. 10, $372(2000)$.

${ }^{5}$ B. J. Alder and T. E. Waiwright, J. Chem. Phys. 27, 1208 (1957).

${ }^{6}$ B. J. Alder and T. E. Waiwright, in Molecular Dynamics by Electronic Computers, edited by I. Prigogine (Transport Processes in Statistical Mechanics, Interscience, New York, 1958), p. 97.

${ }^{7}$ G. A. Bird, Molecular Gas Dynamics and the Direct Simulation of Gas Flows (Clarendon Press, Oxford, United Kingdom,1994).

${ }^{8}$ J. Koplik and J. R. Banavar, Annu. Rev. Fluid Mech. 27, 257 (1995).

${ }^{9}$ Z. Mao and S. B. Sinnott, J. Phys. Chem. 105, 6916 (2001).

${ }^{10}$ Z. Mao and S. B. Sinnott, J. Phys. Chem. 104, 4618 (2000).

${ }^{11}$ M. N. Kogan, in Rarefied Gas Dynamics, edited by L. Trilling (Plenum, New York, 1969).

${ }^{12}$ E. P. Muntz, Annu. Rev. Fluid Mech. 21, 387 (1989).

${ }^{13}$ E. S. Oran, C. K. Oh, and B. Z. Cybyk, Annu. Rev. Fluid Mech. 30, 403 (1998).

${ }^{14}$ G. Karniadakis and A. Beskok, Micro Flows-Fundamentals and Simulation (Springer-Verlag, New York, 2002).

${ }^{15}$ G. Koppenwallner, AGARD Conf. Proc. 11, 1 (1987).

${ }^{16}$ K. A. Fiscko and D. R. Chapman, Proceedings of the 16th International Symposium on Rarefied Gas Dynamics, (AIAA, Pasadena, CA, 1988), p. 374.

${ }^{17}$ X. Zhong, Ph.D. Thesis, Stanford University, Stanford, 1991.

${ }^{18}$ R. K. Agarwal, K. Yun, and R. Balakrishnan, Phys. Fluids 13(10), 3061 (2001).

${ }^{19}$ D. Burnett, Proc. London Math. Soc. 40, 382 (1935).

${ }^{20}$ K. A. Comeaux, D. R. Chapman, and R. W. MacCormack, AIAA Paper No. 95-0415, (1995).

${ }^{21}$ V. P. Sokhan, D. Nicholson, and N. Quirke, J. Chem. Phys. 115(8), 3878 (2001).

${ }^{22}$ K. P. Travis and K. E. Gubbins, J. Chem. Phys. 112(4), 1984 (2000).

${ }^{23}$ K. C. Pong, C. Ho, J. Liu, and Y. Tai, Appl. Microfabrication Fluid Mech. FED 197, 51 (1994).

${ }^{24}$ C. S. Chen, S. M. Lee, and J. D. Sheu, Numer. Heat Transfer, Part A 33, 749 (1998).

${ }^{25}$ J. Pfahler, J. Harley, H. Bau, and J. Zemel, Symposium of Micromechanical Sensors, Actuators, and Systems (ASME DSC 32, New York, 1991), p. 49.

${ }^{26}$ S. B. Choi, R. F. Barron, and R. O. Warrington, Symposium of Micromechanical Sensors, Actuators, and Systems (ASME DSC 32, New York, 1991), p. 123.

${ }^{27}$ J. C. Harley, Y. Huang, and H. Bau, J. Fluid Mech. 284, 257 (1995).

${ }^{28}$ E. B. Arkilic, Ph.D. Thesis, MIT, 1997.

${ }^{29}$ P. H. Nelson and S. M. Auerbach, J. Chem. Phys. 110, 9235 (1999).

${ }^{30}$ D. S. Sholl and K. A. Fichthorn, J. Chem. Phys. 107, 4384 (1997).

${ }^{31}$ D. S. Sholl, Chem. Eng. J. 74, 25 (1999).

${ }^{32}$ D. S. Sholl, Ind. Eng. Chem. Res. 39, 3737 (2000).

${ }^{33}$ J. C. Maxwell, Philos. Trans. R. Soc. London 170, 231 (1879). 
${ }^{34}$ M. von Smoluchowski, Ann. der Phys. Chem. 64, 101 (1898).

${ }^{35}$ A. Beskok, (Ph.D. Thesis, Princeton University, NJ, 1996).

${ }^{36} \mathrm{~J}$. T. Oden and E. R. A. Oliveira, Lectures on Finite Element Methods in Continuum Mechanics (The University of Alabama in Huntsville Press, Huntsville, 1976).

${ }^{37}$ S. Roy and A. J. Baker, Numer. Heat Transfer, Part B 33(1), 5 (1998).

${ }^{38}$ S. Roy and B. P. Pandey, J. Plasma Phys. 68(4), 305 (2002).

${ }^{39}$ S. Roy and B. P. Pandey, Phys. Plasmas 9(9), 4052 (2002).

${ }^{40}$ C. L. Gardner, SIAM (Soc. Ind. Appl. Math.) J. Appl. Math. 54, 409 (1994).

${ }^{41}$ R. D. Richtmyer and K. W. Morton, Difference Methods for Initial-Value
Problems, 2nd ed. (Interscience Publishers, Wiley, New York, 1967).

${ }^{42}$ A. P. Li, F. Muller, A. Birner, K. Nielsch, and U. Gosele, J. Appl. Phys. 84, 6023 (1998)

${ }^{43}$ H. Masuda, H. Yamada, M. Satoh, H. Asoh, M. Nakao, and T. Tamamura, Appl. Phys. Lett. 71, 2770 (1997).

${ }^{44}$ X. Q. Nguyen, Z. Broz, F. Vasak, and Q. T. Nguyen, J. Membr. Sci. 91, 65 (1994).

${ }^{45}$ B. A. Cruden and M. Meyyappan, J. Membr. Sci. (submitted).

${ }^{46}$ K. Itaya, S. Sugawara, K. Arai, and S. Saito, J. Chem. Eng. Jpn. 17, 514 (1984). 